\title{
Der Wärmehaushalt periglazialer Hochgebirgsböden - Zusammenhänge zwischen Bodentiefe und Frostwechseln (Nördlicher Tian Shan, Kasachstan)
}

\author{
Henry Munack \& Hilmar SchröDER*)
}

\begin{abstract}
Kurzfassung: Ganzjährige Bodentemperaturmessungen wurden zwischen den Sommern der Jahre 2003 und 2004 an elf verschiedenen Standorten eines Hochgebirgstales im Zailijskij Alatau (Nördlicher Tian Shan, Kasachstan) durchgeführt. Die periglaziale Höhenstufe - hier, an der Nordabdachung des Gebirges in der Höhe von ca. 2.700 bis $3.750 \mathrm{~m}$ ü. NN - sowie deren Überlappungsbereiche mit den sich vertikal anschließenden Höhenstufen wurden zwischen 2.500 und 4.000 m ü. NN mit einer Äquidistanz von 250 Höhenmetern bemessen.

Die Messungen dienen der Untersuchung des Bodenwärmehaushalts periglazialer Böden im Hochgebirge. Hierfür wurden die Höhenlage und die Exposition der Messstandorte, die Messtiefe und andere assoziierte Faktoren erfasst und zueinander in Beziehung gesetzt. Die Ergebnisse der Messungen geben Auskunft über die Häufigkeit und Standortabhängigkeit von Frostwechseln. Hierdurch können Ableitungen zu deren Intensität und morphologischer Wirksamkeit getroffen werden.
\end{abstract}

[Ground heat balance of periglacial soils in high mountains - Relations between depth below surface and freeze-thaw action (Northern Tian Shan, Kazakhstan)]

\begin{abstract}
Ground temperature measurements have been carried out at eleven different sites of the Prokhodnaja valley in the high mountains of the Zailijskij Alatau (Northern Tian Shan, Kazakhstan) between the summers of 2003 and 2004. For this purpose the periglacial zone and adjacent altitudinal zones have been examined between 2,500 and 4,000 m asl with an equidistance of $250 \mathrm{~m}$. The influences of the altitude, the exposure as well as the depth below the earth's surface on the thermal content and condition of periglacial soils have been considered. The measurements provide useful information about the relations between quantity and quality of freeze-thaw action and the parameters mentioned above.
\end{abstract}

Keywords: ground heat balance, ground temperature measurement, freeze-thaw action, periglacial soils, high mountains, zero curtain, Tian Shan

\footnotetext{
* Adresses of authors: H. Munack, H. Schröder, Humboldt-Universität zu Berlin, Geographisches Institut, Unter den Linden 6, D-10099 Berlin. E-Mail: henry.munack@geo.hu-berlin.de, hilmar.schroeder@geo. hu-berlin.de
} 


\section{Einleitung}

Periglaziale Ökosysteme sind bedeutende Indikatoren für kaltklimatische und paläoklimatische Umweltkonstellationen sowie deren Wandel. Noch prägen Periglazialbereiche große Gebietsanteile der Hochgebirge sowie der Polarund Subpolarregionen. Bedeutende Mengen von Verwitterungsschutt, von Lockersedimenten und von Wasser in Form von Eis sind in den periglazialen Höhenstufen der Hochgebirge gespeichert. Besonders die Hochgebirgsregionen unterliegen jedoch bedeutenden Modifikationen durch die weltweite Klimaänderung. Für die vertikale Höhenstufung vieler Hochgebirge können die Auswirkungen des Climate Change direkt benannt werden. Sie reichen von der Verschiebung der ökologischen Höhengrenzen konkreter, dem Ansteigen der Untergrenze periglazialer Formung und der des Permafrostes - über die Verringerung vergletscherter Areale bis hin zum gänzlichen Rückgang des Permafrostes (Haeberli et al. 2006). Weiterhin führt die Erwärmung gefrorenen Lockermaterials zu einer erhöhten Mobilität desselben (KäÄB et al. 2006). Die hiermit verbundenen Gefahren liegen auf der Hand: Hänge werden instabil, die Mächtigkeit der sommerlichen Auftauschicht (active layer) über Permafrost nimmt zu, einst durch Frost konsolidierte Lockersedimente erhalten Mobilitätspotential und natürliche hydrologische Barrieren werden geschwächt. Die Folgen dieser Entwicklungen können sich in verschiedenster Weise zeigen. Murabgänge in gesteigerter Qualität und Quantität, Ausbrüche von Moränenseen aus ihren Seebecken sowie Hangrutschungen und -stürze sind hierbei nur einige, spektakuläre Szenarien (КӒÄв 2005: $220 \mathrm{ff}$.).

Bevor vom klimabedingten Rückgang der Periglazialbereiche in den Hochgebirgen die Rede sein kann, muss aber die Möglichkeit ihrer, zumindest temporären Ausdehnung in Betracht gezogen werden. Zum höhenwärtigen, Vorauseilen' der Gletschergleichgewichtslinie stellen Haeberli \& Maisch (2007) fest: „Zieht man in Betracht, wie langsam Böden entstehen, die Waldgrenze ansteigt oder Wärme in den Unter- grund geleitet wird, so ergibt sich ein mögliches Bild der zukünftigen alpinen Hochgebirgslandschaft mit weit ausgedehnten Schuttzonen bei langsam abschmelzendem Untergrundeis und stellenweise intensiviertem Abtrag durch reduzierte Hangstabilität und häufigere Starkniederschläge und Hochwasser ".

Eine Vielzahl wissenschaftlicher Arbeiten befasst sich mit der Geomorphodynamik (z.B. Passmore et al. 2008), der Klimageschichte (z.B. Marchenko \& Gorbunov 1997), dem Permafrost (z.B. Gorbunov et al. 1999) sowie der periglazialen Formung und den hiermit assoziierten Prozessen (z.B. Gorbunov \& SEVERSKY 1999) im Nördlichen Tian Shan. Die Zahl der Arbeiten, die sich mit dem Wärmehaushalt periglazialer Böden im Hochgebirge beschäftigen, ist vergleichsweise gering. Vor dem Hintergrund des Climate Change im Hochgebirge und seiner Implikationen ist das Verständnis des oberflächennahen Prozessgefüges jedoch grundlegend für diesbezügliche Fortschritte in der Forschung, da der Grenzbereich zwischen Atmosphäre und Lithosphäre die Korrespondenz der beiden Sphären determiniert (КӒÄB et al. 2007).

Der vorliegende Beitrag präsentiert die Ergebnisse empirisch-induktiver Arbeiten zur Analyse der jahreszyklischen Geomorphodynamik in der periglazialen Höhenstufe des Nördlichen Tian Shan. Unter diesem Gesichtspunkt wird der Frage nach den jährlich wiederkehrenden substratinternen thermischen und kinematischen Prozessen des periglazialen Wirkungsgefüges nachgegangen. Hierbei wird ein besonderer Fokus auf die höhenbedingte Quantität und die Formungswirksamkeit von Frostwechseln gelegt.

Die Bildung periglazialer Formen wird durch die ausgeprägten Reliefunterschiede des Hochgebirges wirksam beeinflusst (HarRIS 1988, ANDrÉ 2003). Auf kleinstem Raum ändern sich maßgebende Faktoren wie die Sonneneinstrahlung, der Vegetationsbesatz oder die Morphologie.

Als Untersuchungsgebiet dient das Prochodnaja-Tal im Nördlichen Tian Shan Kasachstans. Das Tal führt vom Gebirgskamm bei ca. $4.000 \mathrm{~m}$ ü. NN hinab in die äolisch überprägten Fußflächen des Gebirges. Es ist in einem rela- 
tiv naturnahen Zustand. Für die Wahl des Untersuchungsraumes sprechen folgende weitere Gründe: Der Nördliche Tian Shan beherbergt eine Vielzahl aktiver und fossiler Periglazialformen, wie z.B. Blockgletscher und großflächige Solifluktionserscheinungen. Die hierfür grundlegenden Umweltfaktoren sind also im Sinne der Fragestellung besonders günstig.

Weiterhin sind das steile Gebirgsrelief und die rezent hohen Hebungsraten des Gebirges herauszustellen. Ungefähr 40 Prozent der IndischAsiatischen Gesamtkonvergenz, das entspricht ca. $20 \mathrm{~mm} \mathrm{a}^{-1}$ Krustenverkürzung, werden hier abgebaut (Burbank et al. 1999). Die beachtlichen lokalen Jahresniederschläge von bis zu $1.500 \mathrm{~mm}$ (Aizen et al. 1996) sowie die expositions- und höhenbedingten Strahlungsunterschiede im Untersuchungsgebiet sind weitere bedeutende Triebkräfte der regionalen Geomorphodynamik.

Für die Bearbeitung der Fragestellung wurde die Bodentemperatur des kaltklimatisch-morphologischen Responssystems an unterschiedlichen Standorten kontinuierlich aufgezeichnet. Die so erhobenen Punktdaten können zueinander in Beziehung gesetzt werden. Sie sollen der Ableitung übertragbarer Aussagen über die Einflüsse der Höhenlage, der Exposition sowie der bemessenen Bodentiefe auf den Bodenwärmehaushalt dienen.

\section{Untersuchungsgebiet}

Der Nördliche Tian Shan teilt sich in den Zailijskij Alatau im Norden und den Kungej Alatau im Süden, die durch die mächtigen Täler der Flüsse Chon Kemin - nach Westen entwässernd - und Chilik - nach Osten entwässernd getrennt werden (Abb. 1). Der Gebirgsknoten, der beide Höhenzüge vereint, ist das Zentrum der rezenten Vergletscherung des Nördlichen Tian Shan. Die höchste Erhebung des Gebirges bildet der Pik Talgar mit 4.973 m ü. NN. Die Mehrzahl der Gipfel der Hauptkette erreicht Höhen von über $4.000 \mathrm{~m}$ ü. NN.

Das Grundgebirge wird durch proterozoische, ordovizische und silurische saure Intrusiva aufgebaut. Die petrographische Variationsbreite des Substrates ist hierdurch gering und somit vorteilhaft für diese Arbeit.

Der Nördliche Tian Shan liegt im Grenzbereich zyklonaler Einflüsse der Westwinddrift und kalter Luftmassen aus W, NW und N (Aizen et al. 1996). Seine Nordflanke bildet eine wirkungsvolle Sperre für Luftmassen aus N und NW und wird somit zur Insel erhöhter Niederschläge in einer sonst (hoch)kontinentalen Region. In den Wintermonaten sorgen feuchtigkeitsarme Kaltluftmassen für Strahlungswetter und geringe Niederschläge. Gegen März gewinnen jedoch westliche Tiefdruckausläufer an Einfluss. Sie bringen Feuchtigkeit und somit günstige Bedingungen für Schneeakkumulation und Gletscherbildung mit sich (AIzEN et al. 1996).

Die periglaziale Höhenstufe des Zailijskij Alatau ist hochgebirgstypisch verortet. Sie deckt sich ungefähr mit dem Bereich zwischen der oberen Waldgrenze und der klimatischen Schneegrenze, da sie im Wesentlichen durch die Ausdehnung einer perennierenden Schneebzw. Eisdecke sowie einer die Solifluktion hemmenden Vegetationsdecke limitiert wird (Troll 1973, Matsuoka 2003).

Da im Prochodnaja-Tal aktive Blockgletscher bis in Höhen von $3.050 \mathrm{~m}$ ü. NN herabreichen, ist für diese Lagen Permafrost in zumindest diskontinuierlicher Form zu erwarten (GoRBUNOv 1978, King 1996, Matsuoka 2003, SchröDER, KoKarev \& Harrison 2005). Inselhafter Permafrost kann aber auch weit unter der Höhenlage seiner durchschnittlichen Untergrenze von ca. $2.700 \mathrm{~m}$ ü. NN vorkommen (MATSUOKA 2003). Die Höhenverteilung des Permafrostes im Zailijskij Alatau nach MARChENKo \& GoRBUNOV (1997) ist Abb. 2 zu entnehmen.

Die Ausprägung der Klimaelemente im Untersuchungsgebiet variiert mit der Jahreszeit und der Höhenlage. Im Jahresmittel der Lufttemperatur (MAAT) werden im Zailijskij Alatau ab $2.700 \mathrm{~m}$ ü. NN negative Werte erreicht. In ebendieser Höhe befindet sich auch die $10^{\circ}-\mathrm{Ju}-$ li-Isotherme. Bei ca. $3.500 \mathrm{~m}$ ü. NN sinken die Jahresdurchschnittswerte unter $-5^{\circ} \mathrm{C}$. Die sommerliche Null-Grad-Isotherme liegt auf ca. $4.200 \mathrm{~m}$ ü. NN (BLAGOVESCHTSCHENSKIJ 1983), während ihr winterliches Pendant sich unter 

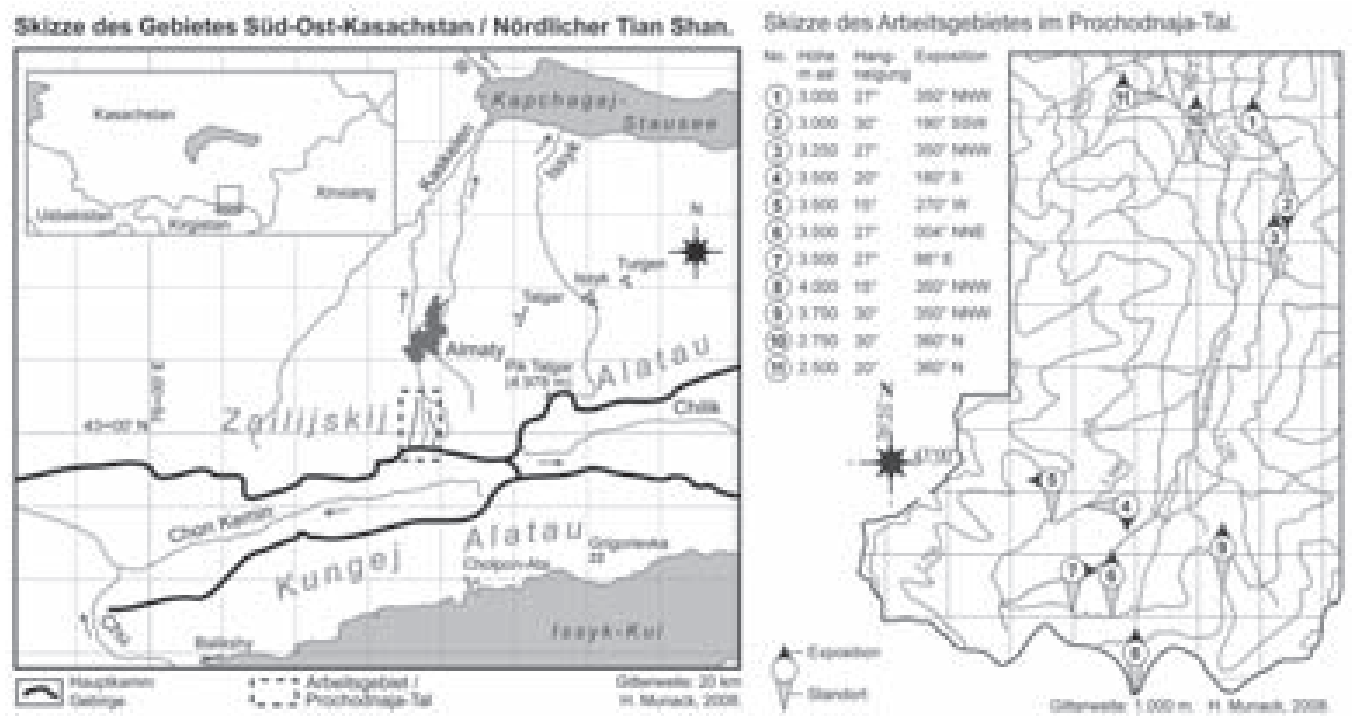

Abb. 1: Skizze Süd-Ost-Kasachstan und Untersuchungsgebiet.

Fig. 1: Spatial situation and sketch of the investigation area.

$1.500 \mathrm{~m}$ ü. NN bewegt (KOKAREV et al. 1997). Die in Tabelle 1 gegebenen Jahresmittelwerte der Lufttemperatur wurden auf Grundlage der von Blagoveschtschenskij (1983) gegebenen Temperaturgradienten sowie der im Messzeitraum auf $3.000 \mathrm{~m}$ ü. NN erhobenen Lufttemperatur (Tab. $13.000 * *$ ) berechnet. Sie decken sich mit den Angaben von BlagoveschtsCHENSKIJ (1983) und KoKAREV et al. (1997) und können somit auch als über den Messzeitraum hinaus repräsentativ betrachtet werden.

Zur Verteilung der Jahresniederschlagssummen im Untersuchungsgebiet stellen BÖHNER \& SCHRÖDER (1999: 27) eine ,,großräumige Zunahme der Niederschläge von SE nach $N W^{\prime \prime}$ fest, „, die im Gebirgsraum durch eine entsprechende expositionsabhängige Differenzierung mesoskalig nachgezeichnet wird“. Die Niederschläge im Zailijskij Alatau nehmen, longitudinal betrachtet, nach Osten sowie nach Westen ab. Mit steigender Höhe nehmen sie zunächst zu (MARCHENKo \& Gorbunov 1997, Aizen et al. 1996). Das Maximum wird mit $1.500 \mathrm{~mm}$ bei ca. 3.400 $m$ ü. NN erreicht. Ab dieser Höhe verhält sich der pluviometrische Gradient rückläufig.
Die Niederschlagswerte variieren aber nicht nur mit der Höhe und der geographischen Lage, sie haben auch eine jahreszeitliche Komponente. Während das Niederschlagsmaximum in den unteren Gebirgslagen bereits im Frühjahr erreicht wird, verlagert es sich in den hochgelegenen Bereichen in den Sommer hinein (Franz 1973, Aizen et al. 1996). Da ,das Auftreten periglazialer Formen und Prozesse ... durch das für die Kryoturbation und Solifluktion wichtige Vorhandensein von Bodenfeuchte während der Frostwechselphasen " bestimmt wird (LeHMKuHL \& Klinge 2000: 75, Schröder, Gunja \& FICKERT 1996), spielt die zeitlich-vertikale Variabilität der Niederschläge für die Formenbildung im Periglazial des Hochgebirges eine entscheidende Rolle. Während der Gefrier- vor allem aber der Auftauphase steht im Untersuchungsgebiet ausreichend Wasser zur Verfügung.

\section{Theorie des Bodenwärmehaushaltes}

Der Bodenwärmehaushalt ist keine statische Größe. Vielmehr umfasst er die „, Größe und Veränderung von Wärmeinhalt und Wärmezu- 

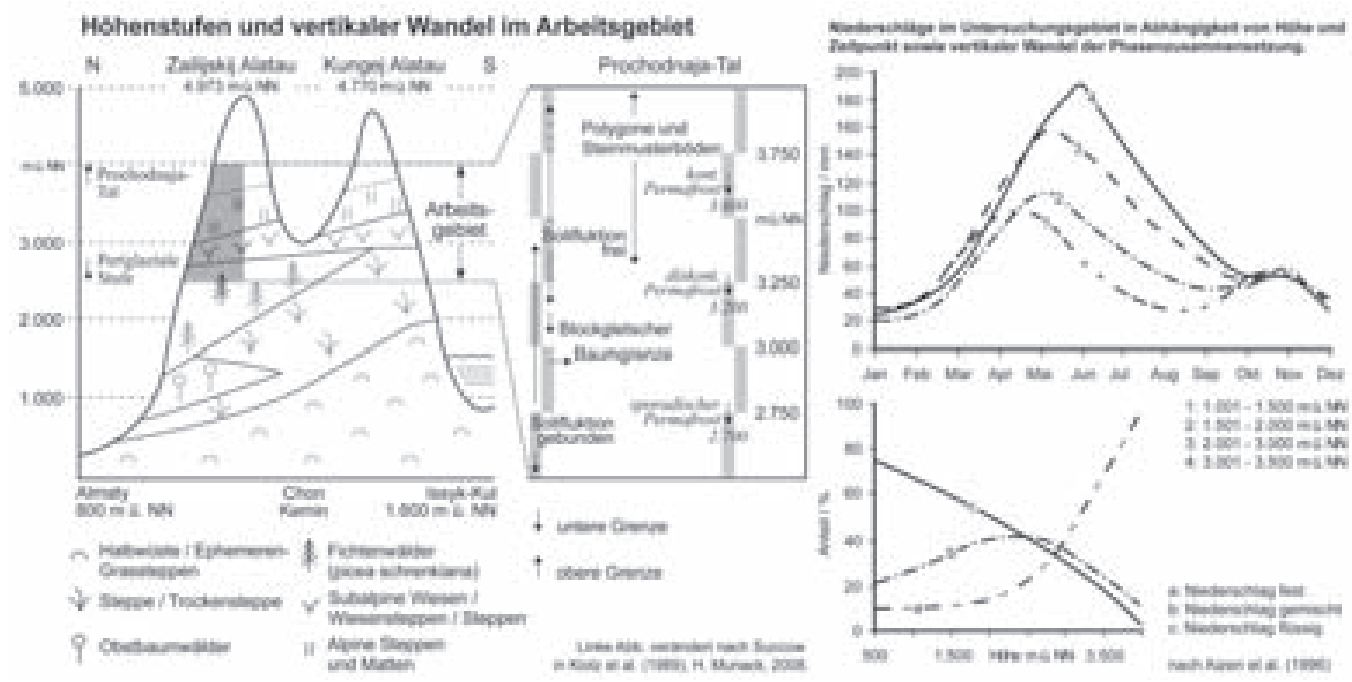

Abb. 2: Höhenstufen der Vegetation, periglaziale Stufe und Niederschläge im Untersuchungsgebiet.

Fig. 2: Altitudinal belts and precipitation in the investigation area.

stand der Böden in Abhängigkeit von Ort, Zeit und Bodentiefe" (ScheFfer \& Schachtschabel 2002: 258) oder, unter stärker physikalischen Prämissen, „sehr typische Temperaturgänge im Boden, gesteuert durch das Zusammenspiel von tages- und jahreszeitbedingtem Wechsel der Einstrahlungsintensität mit Unterschieden von Wärmekapazität ( $c_{w}$ ) und Wärmeleitfähigkeiten ( $\lambda$ ) “(HARTGE \& Horn 1991: 225).

An der Bodenoberfläche, der Grenzfläche zwischen Atmosphäre und Pedosphäre, werden große Energiemengen umgesetzt. Da Ein- und Ausstrahlung über sie stattfinden, ist die Amplitude der Temperaturschwankungen hier naturgemäß am größten. Dies gilt sowohl für die Tagesgänge als auch für die Jahresgänge der Temperatur. Die bedeutendsten Energielieferanten für den Boden sind die kurzwellige solare Strahlung (direkt + diffus) sowie langwellige Strahlungsgewinne aus der Atmosphäre. Die größten Energieverluste entstehen durch die langwellige Abstrahlung und Evaporationsvorgänge. Alle weiteren natürlichen Wärmequellen treten bei durchschnittlich ausgebildetem geothermischem Gradienten in ihrer Bedeutung für den Bodenwärmehaushalt weit hinter die solare Strahlung zurück. Sie finden im Sinne der Fragestellung deshalb keine weitere Beachtung.

Während der Einfluss der Strahlung an der Bodenoberfläche vergleichsweise hoch ist, dringt sie, in Abhängigkeit vom Vegetationsbewuchs und damit der Albedo, aber nur marginal in den Bodenkörper ein. Ihr Einfluss reduziert sich im Boden auf unbedeutende Beträge und ist in wassergesättigtem Granulat ,per Definition gleich Null " (HARTGE \& HoRN 1991: 223). Es bleiben somit zwei wirksame Mechanismen für Wärmeausgleichsbewegungen im Boden: die Wärmeleitung (Konduktion) und die Konvektion. Während die Konvektion von dem Vorhandensein eines mobilen Trägers abhängt, stützt sich die Konduktion auf leitende Querschnitte und ist prinzipiell allgegenwärtig.

Im 3-Phasen-System Boden ist $\lambda$ von der Phasenzusammensetzung abhängig. Eine hohe Wärmeleitfähigkeit hat relativ geringe Temperaturamplituden an der Bodenoberfläche sowie einen vergleichsweise geringen Temperaturgradienten in den oberflächennahen Bodenschichten zur Folge und umgekehrt. Da Wasser eine etwa 20-mal höhere Wärmeleitfähigkeit 
Tabelle 1: Jahresdurchschnittstemperaturen im Prochodnaja-Tal. (Nov. 2003 - Nov. 2004).

Table 1: Mean annual air temperatures in Prokhodnaya valley. (2003-11 - 2004-11).

\begin{tabular}{c|ccccccc}
\hline \hline $\begin{array}{c}\text { Höhe } \\
(\mathrm{m} \text { ü. NN })\end{array}$ & $2.500^{*}$ & $2.750^{*}$ & $3.000^{* *}$ & $3.250^{*}$ & $3.500^{*}$ & $3.750^{*}$ & $4.000^{*}$ \\
\hline MAAT $\left({ }^{\circ} \mathrm{C}\right)$ & 1,3 & $-0,3$ & $-1,8$ & $-3,5$ & $-5,2$ & $-7,1$ & $-8,7$ \\
\hline \hline
\end{tabular}

(*berechnet, ${ }^{* *}$ gemessen)

als Luft besitzt ist $\lambda$ eines Bodens in hohem Maße von seinem Wassergehalt abhängig, zumal Meniskenwasser an Korn-Korn-Kontakten die leitenden Querschnitte signifikant erhöht. Gefriert das Wasser, so steigt die Wärmeleitfähigkeit nochmals um das Vierfache. Dies ist einer der Gründe dafür, dass nicht gefrorenes Wasser in gefrierendem oder bereits gefrorenem Substrat dieses vor rapider Abkühlung schützt (Romanovsky \& Osterkamp 2000). Der Wassersättigungsgrad eines Substrates ist also als entscheidende Komponente für den Wärmehaushalt anzusehen. Er hat sowohl auf $\mathrm{c}_{\mathrm{w}}$ als auch auf $\lambda$ maßgeblichen Einfluss.

Ebenso muss die Bedeutung der Textur (Korngröße, Lagerungsdichte, Korn-Korn-Kontakte) herausgestellt werden, denn silikatische Minerale besitzen noch bessere Wärmeleitungseigenschaften als das Eis. So beträgt die Wärmeleitfähigkeit von Quarz etwa das 3,5-fache der des Eises.

Der Gefrierpunkt des Bodenwassers wird durch gelöste Salze, wenn auch nur minimal, unter $0^{\circ} \mathrm{C}$ gesenkt. Weiterhin führt die zeitweilige Koexistenz von Wasser und Eis im Substrat zum Herabsetzen der Gefriertemperatur. Beim Gefrieren sinkt die Menge flüssigen Wassers, die nun relativ stärker von Kapillar- und Adsorptionskräften beeinflusst wird. Das Wasser gerät unter Spannung und infolge dessen sind für weiteres Gefrieren noch niedrigere Temperaturen vonnöten (Williams \& Sмith 1989, Romanovsky \& Osterkamp 2000). Fällt die Temperatur unter den Gefrierpunkt der freien Bodenlösung wird Kristallisationswärme frei. Für weiteres Gefrieren muss diese freigesetzte Energie abgeführt werden. Wachsende Eiskristalle übernehmen diese Aufgabe sukzessiv, da sie eine geringere $c_{w}$ und ein höheres $\lambda$ als das Wasser besitzen. Während ihres Wachstums nehmen sie Wasser aus ihrer Umgebung auf und entwässern ihr Umfeld. Es bildet sich eine anschwellende Eisschicht. Wird der Punkt erreicht, an dem aus der Umgebung kein Wasser mehr nachgeliefert werden kann, sinkt die $c_{w}$ des Bodens. Die Temperatur des Bodens fällt weiter und auch Wasser, das unter höheren Spannungen steht, kann nun gefrieren. Entspricht die Zufuhr von Wärme dem witterungsbedingten Verlust an der Oberfläche, bildet sich Nadeleis. Ist die Zufuhr jedoch kleiner als der Verlust, so dringt der Frost in den Boden vor (HARTGE \& Horn 1991).

Die oben genannte Gefrierwärme von $332 \mathrm{~J} \mathrm{~g}^{-1}$ wird beim Phasenübergang des Wassers zu Eis frei. Hierdurch kommt es zu bedeutenden Beträgen plötzlich verfügbarer latenter Energie. Eine Folge dessen ist, dass die Bodentemperatur für einen gewissen Zeitraum am Gefrierpunkt stagniert. Dieser sogenannte zero-curtain-Effekt endet, sobald der Großteil des Bodenwassers gefroren ist und eine schnelle Abkühlung in die Tiefe folgt. Der beschriebene Effekt entfaltet seine höchste Wirkung in Böden mit hohen Wassergehalten (Williams \& SмIтH 1989). Beim Auftauen des gefrorenen Bodens kann der umgekehrte Fall des zero curtain eintreten, wobei hierdurch tiefgründige Solifluktionsvorgänge auf längere Zeit effektiv unterbunden werden können (VEIT et al. 1995).

\section{Methoden}

Zur ganzjährigen Aufzeichnung der Bodentemperatur wurden im Sommer 2003 Temperaturlogger in den Boden eingebracht. Die Geräte 
verblieben bis zu ihrer Exhumierung im Herbst 2004 im Substrat. Mit einer Äquidistanz von 250 Höhenmetern wurde somit das Prochodnaja-Tal zwischen 2.500 und $4.000 \mathrm{~m}$ ü. NN bemessen (Abb. 1). Die Obergrenze der Messungen repräsentiert die Kammlage, während die Höhenlage um $2.500 \mathrm{~m}$ ü. NN die Untergrenze der vorgefundenen Periglazialformen markiert. Die Äquidistanz von 250 Höhenmetern soll die Interpretation der Ergebnisse erleichtern. Die 11 bemessenen Standorte wurden - mit Ausnahme des Standortes auf $4.000 \mathrm{~m}$ ü. NN, hier war der Regolith nicht mächtig genug - in Bodentiefen von 5, 15, 30 und $60 \mathrm{~cm}$ bestückt. Die einzelnen Geräte werden im Folgenden nach dem Prinzip 3000n05 [Höhe (3000) - Exposition $(\mathrm{n})$ - Tiefe $(-5 \mathrm{~cm})]$ bezeichnet.

Zur Aufzeichnung der Bodentemperatur wurden Datenlogger der Baureihe HOBO H8-Pro der Firma Onset Computer verwendet. Die erreichte Messgenauigkeit beträgt bei 12-bit-Messungen laut Herstellerangabe $\pm 0,2 \mathrm{~K}$ bei einer maximalen Auflösung von $\pm 0,02$ K. Der interne Speicher erlaubt die Aufzeichnung von 32.645 Messwerten bei Messungen mit der genannten Datentiefe. Der jeweils oberste Datenlogger wurde bewusst nicht direkt an der Erdoberfläche positioniert, um Diebstahl, Abspülung und direkter Insolation vorzubeugen. Die $60 \mathrm{~cm}$ Tiefe für den jeweils untersten Logger stellen hingegen den größten gemeinsamen Nenner für alle Messpunkte dar. Um eine Vergleichbarkeit der Daten zu gewährleisten, wurde die gesamte Messreihe nordexponiert angelegt. Auf der Höhe von 3.500 m ü. NN wurden zusätzlich S-, W- und E-Expositionen belegt, um dem Gewicht von Expositionsunterschieden Rechnung zu tragen. Ein weiterer südexponierter Vergleichsmesspunkt befand sich auf $3.000 \mathrm{~m}$ ü. NN. Als Messintervall wurden 20 Minuten gewählt, da diese Frequenz, bei einem Messzeitraum von einem Jahr und gewünschter 12-bit-Messung dem maximal Möglichen entspricht.

Die Auswahl der Messpunkte erfolgte unter der Prämisse, die Unterschiede zwischen sämtlichen Standortparametern, soweit möglich, gering zu halten. Die Textur sowie die stoffliche Zusammensetzung des Bodenmaterials streuen zwischen den einzelnen Standorten in einem sehr engen Rahmen. Nach der Entfernung des Bodenskeletts wurden die Feinanteile der Bodenproben granulometrisch untersucht. Bis auf den Standort 4000n (Korngrößenmax. im Bereich $\mathrm{mS} \& \mathrm{gS}$ ) konnte hierbei ein jeweiliges Korngrößenmaximum in mittleren Schluffbereich $(\mathrm{mU})$ mit vergleichbaren Summenkurven festgestellt werden. Wir setzen deshalb texturelle Homogenität voraus.

Da nicht für alle Höhenlagen Standorte mit horizontaler Orientierung zu finden waren, fiel die Wahl zugunsten sich ähnelnder Hanglagen aus, zumal sich diese durch höhere Solifluktionsraten auszeichnen. Dies ist im Sinne der Fragestellung erwünscht. Die ausgewählten Standorte unterscheiden sich in ihrer Wölbung nur marginal. Gewisse Standortunterschiede ließen sich trotzdem nicht unterdrücken. So differiert der Wert der Inklination, bei einer mittleren Hangneigung von $24^{\circ}$, zwischen den einzelnen Messpunkten um bis zu $15^{\circ}$ (Abb. 1). Dieser Fehler musste billigend in Kauf genommen werden, da auch nach intensiver Suche keine geeigneteren Standorte gefunden werden konnten.

Bei der Installation der Messreihe wurde verstärkt darauf geachtet, großen Abstand zu Bäumen, Büschen und hohen Gräsern zu halten, um Beschattung und mikroklimatische Störungen zu vermeiden. Für die Messpunkte der montanen und subalpinen Stufe wurden aus diesen Gründen Stellen mit nur spärlicher Vegetationsbedeckung ausgewählt.

Die Messgeräte wurden in die senkrechte Wand der eigens angelegten Gruben eingesetzt. Hierbei wurden die Geräte vertikal gesehen gegeneinander versetzt, um den natürlichen Wärmefluss nicht zu beeinträchtigen. Beachtet werden muss, dass die kürzeste Distanz des Thermistors zur Erdoberfläche mit steigender Hangneigung sinkt, d.h. immer mehr von der lotrechten Entfernung abweicht (Abb. 3). Dies spielt für die oberflächennahen Datenlogger kaum eine Rolle, macht sich ab $30 \mathrm{~cm}$ Tiefe aber durchaus bemerkbar. Dieser Umstand ist bekannt. Die genannten Messtiefen werden aus Gründen der Übersichtlichkeit beibehalten. 
Tabelle 2: Messdauer der Datenlogger (Klassen).

Table 2: Measuring periods of the data loggers.

\begin{tabular}{c|cccccc}
\hline \hline Messdauer & $366 \mathrm{~d}$ & $>300 \mathrm{~d}$ & $>180 \mathrm{~d}$ & $<180 \mathrm{~d}$ & Ausfall & Gesamt \\
\hline Anzahl Logger & 18 & 26 & 32 & 35 & 7 & 42 \\
\hline \hline
\end{tabular}

Die Gruben wurden naturnah verfüllt und gegen Abspülung gesichert. Da es beim Verfüllen nicht zu erheblichen Massenunterschieden kam, kann davon ausgegangen werden, dass das wieder eingebrachte Substrat weder stärker verdichtet noch lockerer lagerte als vor dem Eingraben.

Im Herbst 2004 wurden sämtliche Messpunkte ungestört vorgefunden. Nicht alle Messgeräte haben über den kompletten Messzeitraum Daten aufgezeichnet. Dennoch ist der Prozentsatz der Geräte mit vergleichsweise langen Messreihen mit über 60 Prozent relativ hoch. Ungefähr 75\% der Messungen decken zumindest die Gefrierphase Herbst ab. Circa ein Viertel aller Messungen musste schlussendlich verworfen werden (Tab. 2).

Da beim Einbringen der Messgeräte in den Boden der natürliche Wärmegradient durch das Aufgraben des Substrates gestört wird, wurde eine Karenzzeit von mindestens 14 Tagen zwischen dem Datum des Vergrabens und der ersten verwerteten Messung eingeräumt. Die Messergebnisse belegen jedoch, dass schon die Hälfte der Karenz ausreichend gewesen wäre. Um für den jeweiligen Messpunkt und die einzelnen Messtiefen entscheiden zu können, ob ein Frosttag, Frostwechseltag oder frostfreier Tag vorlag, wurden zunächst das Tagesmaximum und -minimum der Temperaturwerte, gemessen am jeweiligen Datenlogger, gebildet. Der Zuschlag zu einer der drei Klassen erfolgte nach den in Tabelle 3 definierten Regeln.

Der Definition der drei Klassen liegen die eingangs erläuterten physikalischen GesetzmäBigkeiten zugrunde. Aufgrund der negativen Verschiebung der Gefriertemperatur wurden jene Tage, an denen die Maximal- und Minimaltemperatur $0^{\circ} \mathrm{C}$ betrugen, den frostfreien Tagen zugerechnet. Gleiches gilt für Tage mit einem positiven Maximum und einem Minimum von $0^{\circ} \mathrm{C}$. Tagen mit nur unidirektionalem Überschreiten der Gefriergrenze werden im Folgenden zwar unterschiedliche formungsbe-

Tabelle 3: Entscheidungsmatrix Frosttag, frostfreier Tag oder Frostwechseltag.

Table 3: Decision matrix - cryotic day, non-cryotic day or day with freeze-thaw action.

\begin{tabular}{c|c|c|c|l|l}
\hline \hline & Tagesmaximum & & Tagesminimum & & Klasse \\
\hline \multirow{6}{*}{ Wenn } & $>0^{\circ} \mathrm{C}$ & & $>0^{\circ} \mathrm{C}$ & & frostfreier Tag \\
$>0^{\circ} \mathrm{C}$ & & $<0^{\circ} \mathrm{C}$ & & Frostwechseltag \\
& $<0^{\circ} \mathrm{C}$ & und & $<0^{\circ} \mathrm{C}$ & dann & Frosttag \\
& $>0^{\circ} \mathrm{C}$ & & $0^{\circ} \mathrm{C}$ & & frostfreier Tag \\
& $0^{\circ} \mathrm{C}$ & & $0^{\circ} \mathrm{C}$ & & frostfreier Tag \\
& $0^{\circ} \mathrm{C}$ & & $<0^{\circ} \mathrm{C}$ & & Frosttag \\
\hline
\end{tabular}




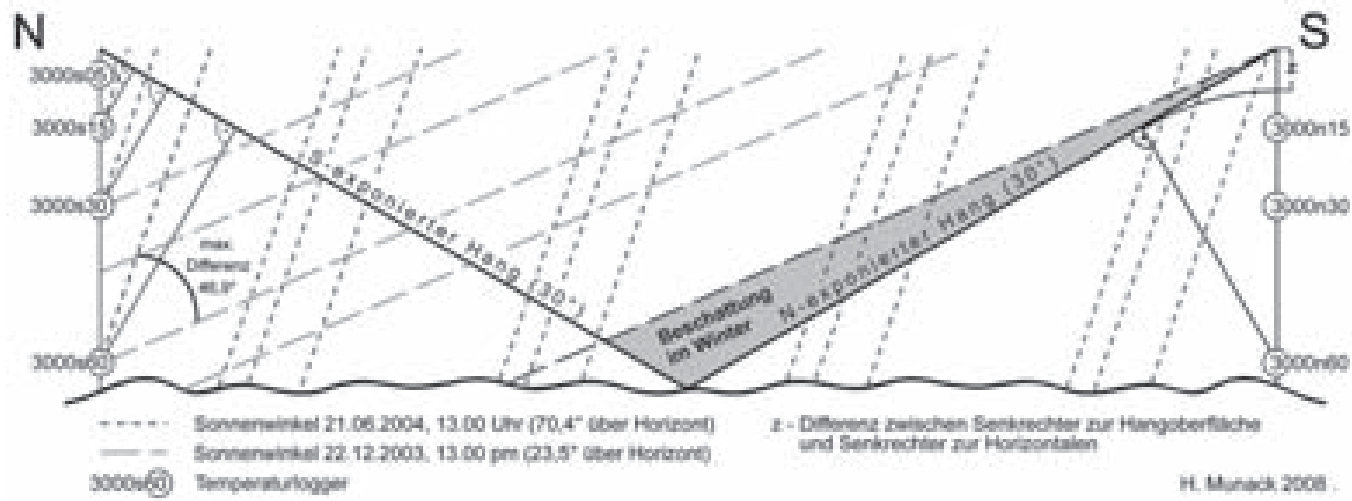

Abb. 3: Zusammenspiel von Exposition, Hangneigung, Strahlungswinkel und Beschattung.

Fig. 3: Interaction of exposure, inclination and insolation.

zogene Wertigkeiten zugesprochen (МАтSUокA $2003 \& 2005$ ), sie alle müssen aber als Frostwechsel gesehen werden. Im Jahreszyklus der bemessenen Höhenlagen gehört zu jedem Frostereignis, wenn auch mit einigen Monaten Zeitunterschied, eine Auftauphase. Es ist also ein Frostwechsel gegeben, wenn auch mit großer Zwischenzeit.

\section{Ergebnisse und Diskussion}

Die gemessenen Werte reichen von minimal $-20,1{ }^{\circ} \mathrm{C}$ bei Messpunkt $4000 \mathrm{n} 50$ bis zu maximal 18,54 C an Messpunkt 3500n05. Die niedrigste Jahresmitteltemperatur wurde mit $-6,93$ ${ }^{\circ} \mathrm{C}$ ebenfalls an Messpunkt 4000n50 aufgezeichnet. Der höchste Durchschnittswert konnte an Messpunkt $3000 \mathrm{~s} 60$ mit $3,44^{\circ} \mathrm{C}$ gemessen werden. Es ist vorstellbar, dass am Standort 2500n höhere Durchschnittswerte erreicht wurden. Hierfür gibt es durch oben genannte Ausfälle der Messgeräte allerdings keinen Beleg. Die Spannweiten zwischen den Maximalund Minimalwerten der einzelnen Messpunkte schwanken zwischen 7,99 $\mathrm{K}$ bei Messpunkt 3000n60 und 26,36 K am Messpunkt 3500n05.

\subsection{Einfluss der Bodentiefe auf Wärmeinhalt und -zustand des Bodens}

Wie zu erwarten war, lässt sich aus den Temperaturmessungen ein mit zunehmender Boden- tiefe schwindender atmosphärischer Einfluss ableiten. Sowohl die Tages- als auch die Jahresamplitude der Bodentemperatur verringern sich mit zunehmender Tiefe unter der Erdoberfläche, die Temperaturkurven erscheinen geglättet und die Wahrscheinlichkeit von ,Ausreißern' sinkt. Generell lässt sich somit ein Wandel von einem tageszeitlichen $\mathrm{zu}$ einem jahreszeitlichen Frostwechselklima mit zunehmender Bodentiefe feststellen.

$A b b .4$ bildet diesen Sachverhalt exemplarisch für die Temperaturverläufe der Messpunkte 3000n und 3000s im Zeitraum vom 09. Juni bis 12. Juni ab. Das Tagesmaximum der Temperatur wird in $15 \mathrm{~cm}$ Tiefe erst ca. $6 \mathrm{~h}$ später erreicht als in $5 \mathrm{~cm}$ Bodentiefe. Zu diesem Zeitpunkt ist in $60 \mathrm{~cm}$ Bodentiefe gerade das Temperaturminimum erreicht. Am nordexponierten Standort treten in $60 \mathrm{~cm}$ Tiefe keine Temperaturschwankungen mehr auf und auch am südexponierten Messpunkt 3000s60 sind keine starken Schwankungen mehr festzustellen. Dennoch lässt sich hier noch eine geringe Tagesamplitude nachweisen. Eben genannte Differenzen zwischen den Messpunkten 3000n60 und 3000s60 gehören in die Diskussion um die Auswirkung von Expositionsunterschieden.

Bei der Betrachtung des Jahrestemperaturverlaufes ist ein noch viel größerer TemperaturZeit-Versatz zwischen den einzelnen Bodentiefen zu erkennen. So liegen z.B. fast zwei Monate zwischen der Unterschreitung der 


\section{Vergleich der Standorte 3000n und 3000s, 09, bis 12. Juni 2004}

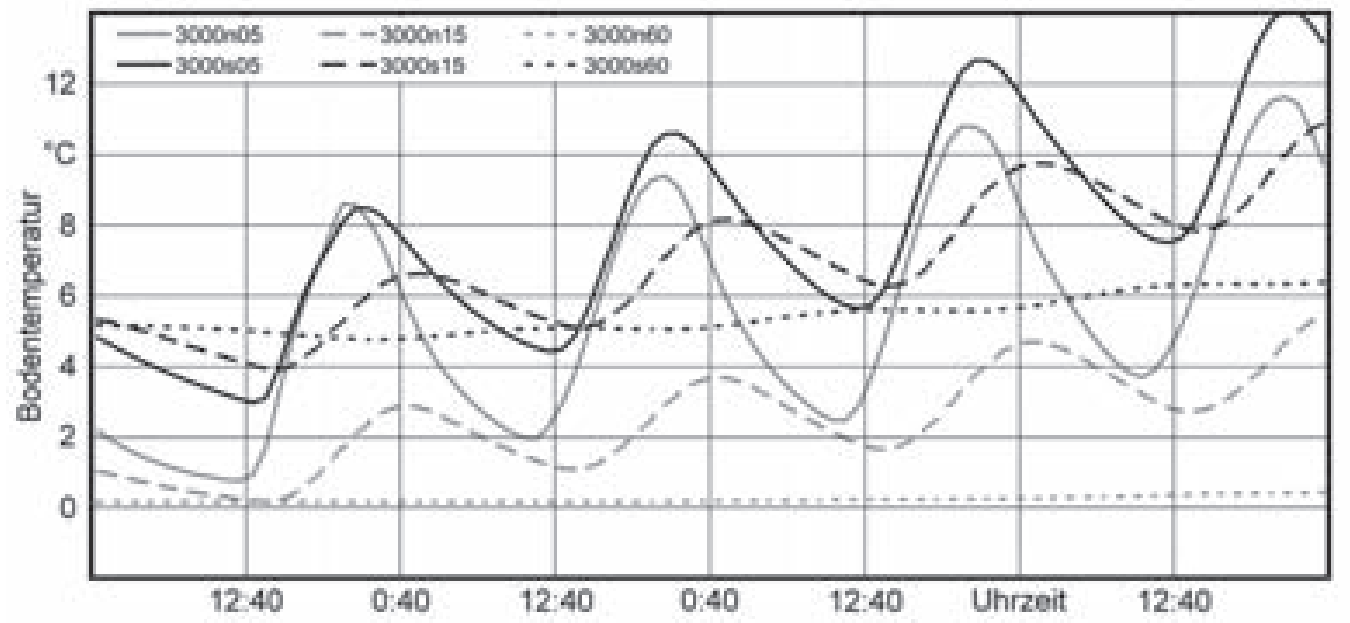

Abb. 4: Tagesvergleich der Standorte 3000n und 3000s.

Fig. 4: Comparison of measuring sites 3000n and 3000s.

0-Grad-Schwelle an Messpunkt 3000n05 und 3000n30 (Abb. 5). In $60 \mathrm{~cm}$ Tiefe wird hier zu keinem Zeitpunkt ein negativer Temperaturwert aufgezeichnet. Die Gründe hierfür sind in der Wärmeleitfähigkeit des Substrates und in dem anliegenden Luft-Boden-Gradienten der Temperatur zu suchen. Als andere, nicht materialspezifische Ursachen sind die Auswirkung einer Schneedecke auf den Bodenwärmehaushalt und insbesondere der Zeitpunkt ihres Eintreffens zu nennen.

Die in Abb. 5 präsentierten Ergebnisse lassen folgende erste Aussagen zu: Die Zahl der Frosttage nimmt mit der Höhe zu. Der Abschnitt der Frosttage öffnet sich mit steigender Höhe trichterförmig. Der Unterschied zwischen $3.000 \mathrm{~m}$ und $4.000 \mathrm{~m}$ ü. NN drückt sich, in einem halben Meter Bodentiefe, durch 300 zusätzliche Tage mit negativen Temperaturwerten aus. Die nordexponierte Lage auf $3.000 \mathrm{~m}$ ü. $\mathrm{NN}$ ist in $60 \mathrm{~cm}$ Tiefe zu keinem Zeitpunkt gefroren. Die gemessenen Frostwechseltage im Boden stehen zahlenmäßig weit hinter den Frostwechseltagen der Luft zurück. An vielen Standorten beschränken sich die Frostwechselereignisse auf das einmalige jährliche Unter- und Wiederüberscheiten des Nullpunktes. Von 5 auf $60 \mathrm{~cm}$
Bodentiefe halbiert sich die Spannweite der Jahrestemperaturamplitude ungefähr.

\subsection{Frostwechsel}

Die Anzahl der Frostwechsel ist im gesamten Vertikalprofil überraschend gering. Allerdings muss hierbei beachtet werden, dass oberflächennahe Bereiche in Höhen ab $3.500 \mathrm{~m}$ auch in den Sommermonaten nächtlichem Gefrieren ausgesetzt sind. Bei längeren Geländeaufenthalten in den Monaten Juli, August und September der Jahre 2003 und 2004 wurden in den Morgenstunden täglich 20 bis $40 \mathrm{~mm}$ starke Kammeislagen vorgefunden, deren formungsaktive Wirksamkeit unter anderem bei LEHMKUHL und KLINGE (2000) beschrieben wird. Diese Frostwechselaktivitäten wurden jedoch schon von den in $5 \mathrm{~cm}$ Tiefe positionierten Datenloggern nicht mehr erfasst. Die Zahl der Frostwechseltage an der Grenzschicht zwischen Atmosphäre und Boden dürfte also um einiges höher liegen als mit den Loggermessungen ermittelt. Matsuoka (2003) schildert diese vergleichsweise hochfrequenten GefrierAuftau-Vorgänge, denen er die Bildung flachgründiger, kleinteiliger periglazialer Formen 


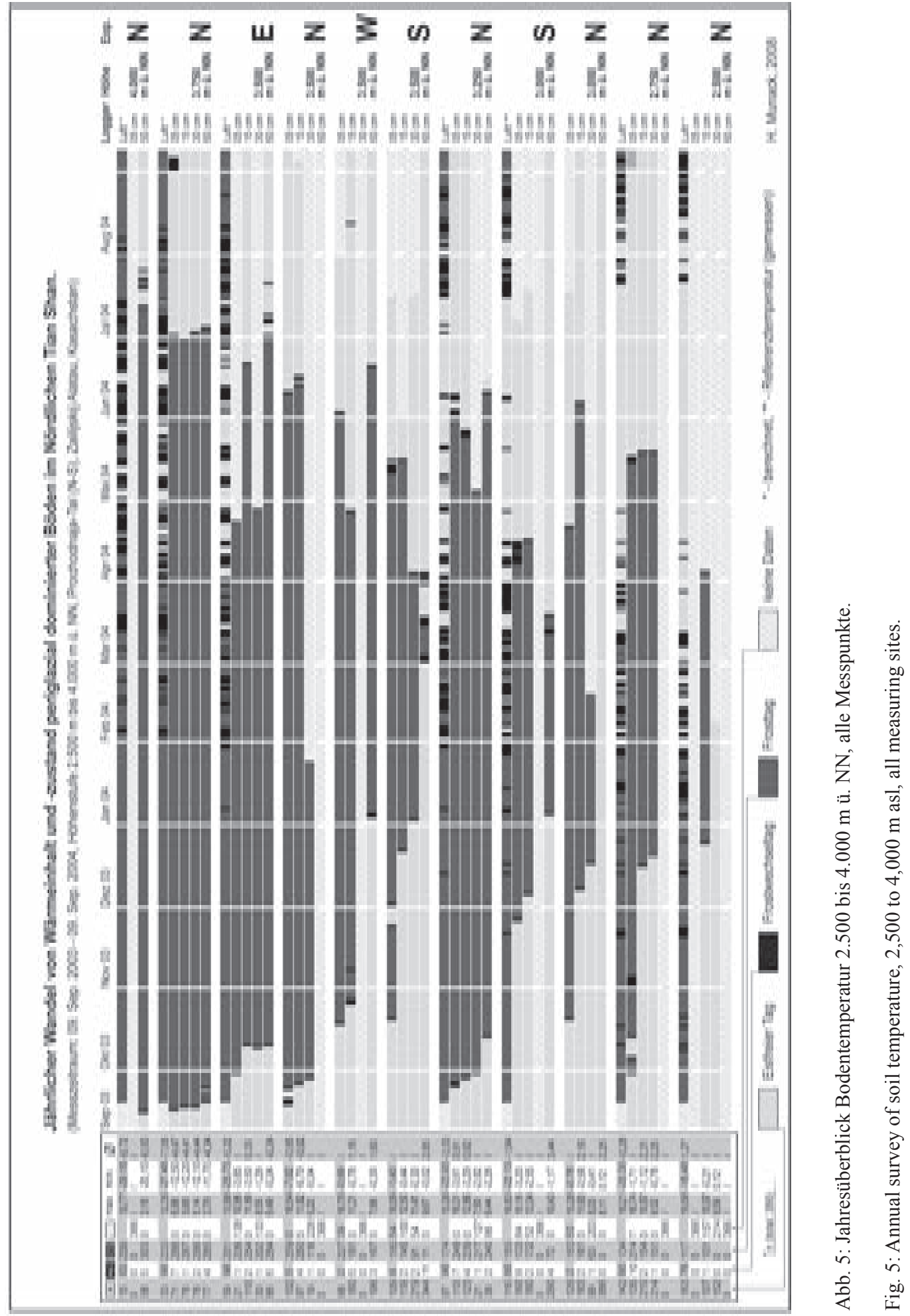




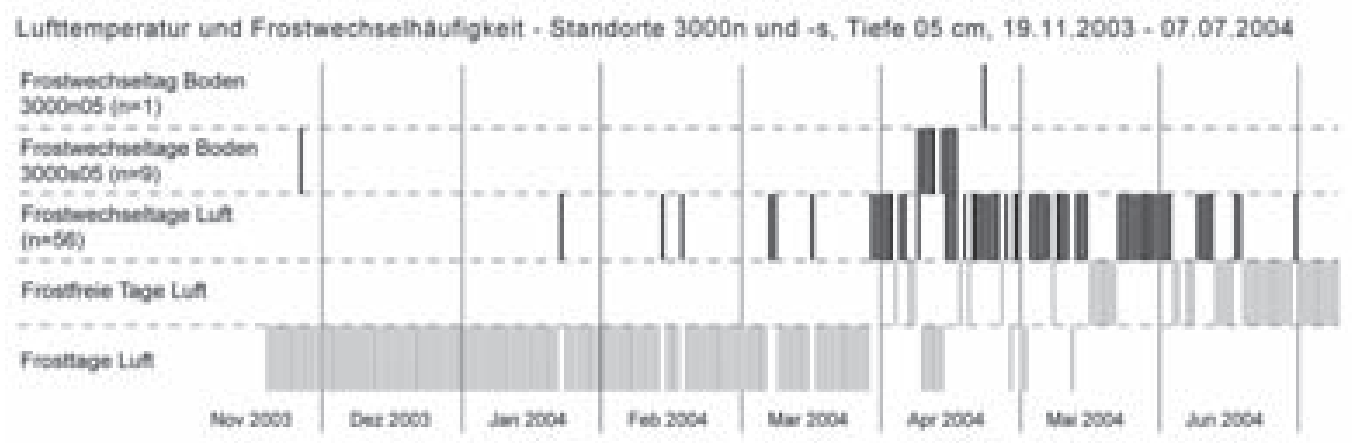

Abb. 6: Vergleich der Frostwechselhäufigkeit Luft und Boden, $3.000 \mathrm{~m}$ ü. NN.

Fig. 6: Comparison of the freeze-thaw action air/ground at $3000 \mathrm{~m}$ asl.

und Steinmuster zuschreibt. Zeitgleiche Beobachtungen oberflächennaher Bereiche zeigen „,...that such shallow and rapid movements result mainly from needle ice creep and diurnal frost creep enhanced by periodic precipitation " (Матsuока 2003: 159). Durch Frostwechsel induzierte Bewegungsraten können somit als Funktion der Frostwechselhäufigkeit, der Mächtigkeit der betroffenen Ablagerungen und der Hangneigung betrachtet werden (MATSuOKA 1998).

In Abb. 6 werden die Frostwechselhäufigkeiten der Messpunkte 3000n05 und 3000s05 jenen der Luft, ebenfalls auf $3.000 \mathrm{~m}$ ü. NN gemessen, gegenübergestellt. Zuerst fällt auf, dass das Verhältnis der Frostwechsel im Boden zwischen nord- und südexponierter Lage 1:9 beträgt. Die Gründe hierfür liegen in der erhöhten Strahlungsgunst der südexponierten Lage. Während der Auftauphase im Frühjahr 2004 (07.-15.04.) kam es hier, im Gegensatz zur Nordlage, zu täglichen Frostwechselvorgängen mit steigender Länge (Abb. 7).

Das Verhältnis der Frostwechsel des Standortes 3000s05 (Boden) zur Luft beträgt nochmals 9:56 (entspr. 1:6), das des Standortes 3000n05 (Boden) zur Luft sogar 1:56. 56 Frostwechseltage der Lufttemperatur stehen also einem Frostwechseltag in $5 \mathrm{~cm}$ Bodentiefe gegenüber. Hier wird deutlich, wie verzögert die Bodentemperaturen auf Veränderungen der Lufttemperatur reagieren. Ephemere atmosphärische Frostwechselereignisse haben augenscheinlich schon auf Bodentiefen von nur $5 \mathrm{~cm}$ keinen Einfluss mehr. Frostverwitterung und -sprengung sind demnach offensichtlich Vorgänge, die im Untersuchungszeitraum keine tägliche Frequenz besitzen, da die Lufttemperatur im Rahmen durchschnittlicher Nachtfröste nicht schnell und tief genug fällt, um einen genügend starken Temperaturgradienten in den Boden auszubilden.

Aus den Messdaten können bestimmte raumzeitliche Muster des Auftretens von Frostwechseln abgeleitet werden. Die Aussagen beziehen sich auf den Untersuchungsraum zum Zeitpunkt der Untersuchung.

In der Regel sind die Frostwechsel im Boden an den Beginn der jährlichen Gefrier- bzw. Auftauphase gebunden, d.h. jahreszeitlich bedingte Phasen von Gefrornis und Frostfreiheit werden nicht durch Frostwechsel unterbrochen.

Mit zunehmender Bodentiefe lässt sich ein Wandel von ephemeren zu annuellen Frostwechseln feststellen. Schon ab einer Bodentiefe von $15 \mathrm{~cm}$ beschränkt sich die Zahl der Frostwechsel i.d.R. auf das einmalige Unterschreiten und zwangsläufig spätere Überschreiten des Gefrierpunktes. Dies geschieht jeweils ein Mal im Jahr, zu Beginn und zum Ende der Gefrierphase.

Auf südexponierten Hängen kommt es häufiger zu Frostwechseln als auf nordexponierten. Die Gründe hierfür sind die zeitweilige direkte Besonnung sowie die höheren Durchschnittstemperaturen (Abb. 5). Eine weitere Rolle spielt 


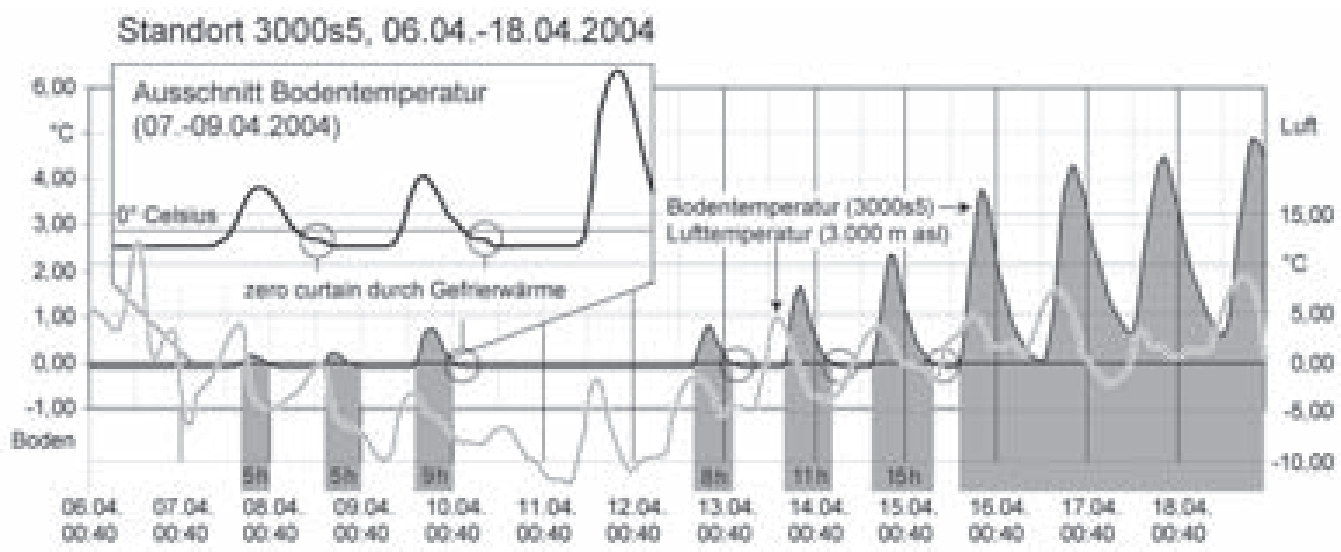

Abb. 7: Frostwechsel am Standort 3000s.

Fig 7: Freeze-thaw action at site 3000s.

hier mit Sicherheit die geringere Schneebedeckung der Südseiten durch deren Niederschlagsungunst (Aızen et al. 1999) sowie das zeitlich frühere Ausapern dieser Lagen. Zur Mächtigkeit und Dauer der Schneebedeckung im Untersuchungszeitraum liegen leider keine Daten vor.

Mit steigender Höhe verschieben sich die winterlich-frühjährlichen Frostwechsel in die Sommerzeit hinein. Dies begünstigt die morphologische Wirksamkeit der Frostwechsel, da sie zeitgleich mit den stärksten Niederschlägen auftreten.

Herbstliche Frostwechsel treten in tieferen Lagen zeitlich später als in höheren Bereichen auf. Durch die erhöhte Wahrscheinlichkeit einer bereits vorhandenen geschlossenen Schneedecke werden herbstliche Frostwechsel in tieferen Lagen morphologisch vergleichsweise weniger wirksam.

Weiterhin muss festgestellt werden, dass ein Großteil der erfassten Frostwechsel zwar den Nullpunkt unterschritten hat, die Temperaturen aber sicher nicht zum Gefrieren größerer Mengenanteile von Bodenwasser geführt haben dürften. Die durchgezogene Kurve in Abb. 7 bildet den Temperaturverlauf in $5 \mathrm{~cm}$ Bodentiefe ab. Es kam im abgebildeten Zeitraum zu keiner Abkühlung unter $-0,13^{\circ} \mathrm{C}$, obwohl die Lufttemperatur im gezeigten Zeitraum stellenweise ganz erheblich unter den Gefrierpunkt sank.
Da das sechsfache Eindringen der Gefrierfront bis zur immergleichen Temperatur höchst unwahrscheinlich ist, muss eine andere Erklärung gefunden werden, zumal zwischen den Frostwechseltagen ein Tag völliger Stagnation liegt. Offensichtlich liegt hier zero curtain vor. Die freigesetzte Wärme beim Phasenübergang von Wasser zu Eis verhindert ein Absinken der Temperatur. Es kann somit für diesen Standort und Zeitpunkt eine initiale Gefriertemperatur von minimal $-0,13^{\circ} \mathrm{C}$ angenommen werden.

Da die Tageskurven der Temperatur nicht symmetrisch sind - bevor die Temperatur unter $-0,1^{\circ} \mathrm{C}$ sinkt verbleibt sie für ca. 1 Stunde auf diesem Niveau (Abb. 7) - lässt sich der Temperaturpunkt beginnender Gefriervorgänge sogar noch genauer (auf ebenjenen Wert) beziffern. In nur zehn Zentimeter tieferen Bereichen schwankt die Temperatur überhaupt nicht mehr. Sie stagniert in dieser Tiefe bei $-0,05^{\circ} \mathrm{C}$, was nach eben Gesagtem dem Auftauzustand bzw. einem ausklingenden zero curtain entspricht.

Für die Darstellung der Frostwechselhäufigkeiten (Lufttemperatur) in Abb. 8 und Abb. 9 wurde die schon eingeführte Entscheidungsmatrix (Tab. 3) verwendet. Als Datengrundlage dienen Temperaturgradienten nach Blagoveschtschenskij (1983) und unveröffentlichte Lufttemperaturwerte der Akademie der Wissenschaften Kasachstans, gemessen auf $3.000 \mathrm{~m}$ ü. NN. 


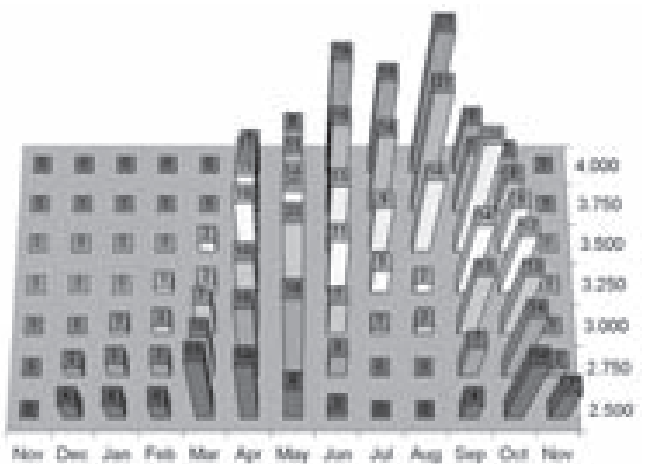

Abb. 8: Frostwechseltage Luft in Abhängigkeit von Zeit und Höhe ü. NN (n Frostwechseltage $=$ Zahl auf Säule).

Fig. 8: Days with freeze-thaw action depending on date and altitude; $\mathrm{n}$ freeze-thaw days $=$ figure on column.

Die Zahl der Frosttage steigt mit der Höhe, während Anteil der frostfreien Tage stark zurückgeht. Bei ca. $2.800 \mathrm{~m}$ ü. NN halten sich Frosttage und frostfreie Tage zahlenmäßig ungefähr die Waage. In dieser Höhe verhalten sich ebenfalls die Anteile flüssigen und festen Niederschlages (Abb. 2) äquivalent. Des Weiteren sind hier folgende vertikale Übergangsbereiche zu finden: die Waldgrenze, die Untergrenze diskontinuierlichen Permafrostes sowie die gemittelte jährliche Null-Grad-Isotherme.

Auf $4.000 \mathrm{~m}$ ü. NN werden nur noch 15 Tage mit ausschließlich positiven Tagestemperaturen erreicht. Die Zone der meisten Frostwechsel liegt bei ca. $3.500 \mathrm{~m}$ ü. NN. Ihre Anzahl sinkt mit fallender sowie mit steigender Höhe. Die absolute Anzahl der Frostwechsel dürfte sogar noch höher liegen als angegeben, da es sich um Frostwechseltage handelt und somit extrem kurzfristige Gefrier-Auftau-Vorgänge als Tagesereignis zusammengefasst werden. In den unteren Lagen bis $3.250 \mathrm{~m}$ ü. NN sind eindeutig zwei Phasen der Frostwechselaktivität zu erkennen. Sie liegen im Frühjahr und im Herbst. Mit steigender Höhe ü. NN verschieben sich die Frostwechselphasen in den Sommer hinein und aus der bimodalen wird eine unimodale Verteilung (Abb. 8).

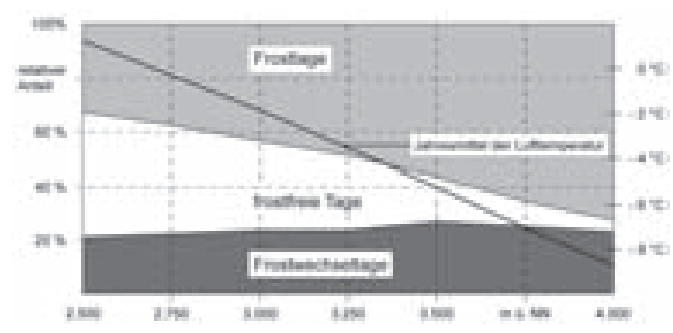

Abb. 9: Vertikaler Wandel des Verhältnisses Frosttage - frostfreie Tage - Frostwechseltage im Untersuchungszeitraum.

Fig. 9: Vertical changing relation of cryotic, noncryotic and freeze-thaw days.

\section{Schlussfolgerungen}

Da sich große Permafrostbereiche der Hochgebirge thermisch im Bereich nahe des Gefrierbzw. Schmelzpunktes befinden, haben bereits geringe atmosphärische Änderungen einen Einfluss auf ihre Mobilität. Erhebliche Lockermassen in Steillagen sowie weite Hangbereiche sind dadurch in ihrer Stabilität gefährdet. Hierin besteht ein großes Gefahrenpotential für die betroffenen Regionen.

Die vorliegende Arbeit beschreibt das jährliche Wechselspiel von Frostphasen und frostfreien Phasen im Hochgebirge des Nördlichen Tian Shan. Es konnte gezeigt werden, dass sich die eingeschalteten Frostwechselphasen in der Regel mit der jährlichen Gefrier- bzw. Auftauphase decken. Die formungsaktive Wirksamkeit einzelner, kurzfristiger atmosphärischer Frostwechsel darf hierbei nicht überschätzt werden. Bereits Bodentiefen von $5 \mathrm{~cm}$ unterliegen nicht mehr dem täglichen atmosphärischen FrostwechselRegime. Es konnte, für einen ausgewählten Zeitraum, ein Verhältnis der Frostwechselhäufigkeit von 1 (Boden, nordexponiert) zu 9 (Boden, südexponiert) zu 56 (Luft) nachgewiesen werden. Unter Beachtung der örtlichen Niederschlagsverteilung (Abb. 2) lässt sich aus den Messungen ableiten, dass in den Höhenlagen zwischen 
ca. $3.500 \mathrm{~m}$ und $3.750 \mathrm{~m}$ ü. NN die Bedingungen für den Ablauf periglazialer Prozesse, die Frostwechsel und Feuchte voraussetzen, am günstigsten sind. In diesen Höhenbereichen sind mächtige Schuttkörper zu finden. Viele davon befinden sich in Steillagen oder sind durch den (sub)rezenten Abbau von Gletschern freigelegt worden. Die Bewertung dieses Gefahrenpotentials ist eine große Herausforderung der nahen Zukunft für die Geowissenschaften.

\section{Acknowledgement}

We thank Sergey Kurashkin, Irina Shesterova and Alexander Kokarev for hosting us and for administrative assistance. Mario Fest and Andreas Wille greatly helped with the fieldwork. HM thanks DAAD (Deutscher Akademischer Austauschdienst) for travel grant during the first field season. The paper considerably benefited from the constructive comments of two anonymous reviewers, who are gratefully acknowledged.

\section{Literatur}

Aizen, W., Aizen, E. \& Melack, J. (1996): Precipitation, melt and runoff in the northern Tien Shan. - Journal of Hydrology, 186: 229-251. DOI: 10.1016/S0022-1694(96)03022-3.

ANDRÉ, M.-F. (2003): Do periglacial landscapes evolve under periglacial conditions? - Geomorphology, 52: 149-164. DOI: 10.1016/S0169555X(02)00255-6.

BlagoveschtschenskiJ, W.P. (1983): Das Temperaturregime der hochgelegenen Gebiete Zentralasiens und Kasachstans. - In: AKADEMIE DER WISS. DER KASACHISCHEN SSR (Hrsg.): Gletscher, Schneedecken und Lawinen der Gebirgsregionen Kasachstans. Ledniky, sneschnij pokrow i lawiny gornich rayonow Kasachstana); Alma Ata (in russisch).

BÖHNER, J. \& SCHRÖDER, H. (1999): Zur aktuellen Klimamorphologie des Tienschan. - Petermanns Geographische Mitteilungen, 143: 17-32.

Burbank, D.W., McLean, J.K, Bullen, M.E., Abdrakhmatov, K.Y. \& Miller, M.M. (1999): Partitioning of intermontane basins by thrustrelated folding, Tien Shan, Kyrgyzstan. - Basin Research, 11: 75-92. DOI: 10.1046/j.13652117.1999.00086.x.
Franz, H.-J. (1973): Physische Geographie der Sowjetunion. -535 S.; Gotha (Haack).

Gorbunov, A., Marchenko, S. \& Seversky, E. (1999a): The thermal environment of blocky materials in the mountains of Central Asia. Permafrost and Periglacial Processes, 15/1: 9598. DOI: $10.1002 / \mathrm{ppp} .478$.

Gorbunov, A. \& Seversky, V. (1999): Solifluction in the Mountains of Central Asia: Distribution, Morphology, Processes. - Permafrost and Periglacial Processes, 10: 81-89.

Gorbunov, A. (1978): Permafrost Investigations in High-Mountain Regions. - Arctic and Alpine Research, 10/2: 283-294.

Haeberli, W. \& Maisch, M. (2007): Klimawandel im Hochgebirge. - Der Klimawandel - Einblicke, Rückblicke und Ausblicke: 98-107.

Haeberli, W., Cheng Guodong, Gorbunov, A.P. \& Harris, S.A. (2006): Mountain permafrost and climatic change. - Permafrost and Periglacial Processes, 4/2: 165-174. DOI: 10.1002/ ppp.3430040208.

Harris, S. A. (1988): The Alpine Periglacial Zone. - In: Clark, M. J. (ed.): Advances in Periglacial Geomorphology: 369-413.

Hartge, K. H. \& Horn, R. (1991): Einführung in die Bodenphysik, 3. Aufl. - 304 S.; Stuttgart (Enke).

Hintermaier-ERHARD, G. \& ZeCh, W. (1997): Wörterbuch der Bodenkunde. -338 S.; Stuttgart (Enke).

KäÄB, A., Chiarle, M., Raup, B \& Schneider C. (2007): Climate change impacts on mountain glaciers and Permafrost. - Global and Planetary Change, 56/1-2: vii-ix. DOI: doi:10.1016/j.gloplacha.2006.07.008.

Kä̈̈̈, A., Frauenfelder, R. \& Roer, I. (2007a): On the response of rockglacier creep to surface temperature increase. - Global and Planetary Change, 56/1-2: 172-187. DOI: 10.1016/j.gloplacha.2006.07.005.

KÄÄB, A. (2005): Remote sensing of Mountain Glaciers and Permafrost Creep. - Schriftenreihe Physische Geographie. Glaziologie und Geomorphodynamik. 48: $266 \mathrm{~S}$.

KING, L. (1996): Dauerfrostboden im Gebiet Zermatt - Gornergrat - Stockhorn: Verbreitung und permafrostbezogene Erschließungsarbeiten. - Zeitschrift für Geomorphologie, NF, Suppl.-Bd. 104: 73-93.

KLotz, G. (Hrsg.) (1989): Hochgebirge der Erde und ihre Pflanzen- und Tierwelt. - 355 S.; Leipzig (Urania-Verlag).

Kokarev, A., Shesterova, I. \& SchröDer, H. (1997): Die Blockgletscher im Sailijskij Alatau des Ti- 
enschan. - Hallesches Jahrbuch der Geowissenschaften, 19: 81-94.

LehmKuhl, F. \& Klinge, M. (2000): Bodentemperaturmessungen aus dem Mongolischen Altai als Indikatoren für periglaziale Geomorphodynamik in hochkontinentalen Gebirgsräumen. - Zeitschrift für Geomorphologie, NF, 44/1: 75-102.

Marchenko, S. \& Gorbunov, A. (1997): Permafrost Changes in the Northern Tien Shan during the Holocene. - Permafrost and Periglacial Processes, 8: 427-435. DOI: 10.1002/ (SICI) 1099-1530(199710/12)8:4<427::AIDPPP262>3.0.CO;2-V.

MatsuoKa, N. (2005): Temporal and spatial variations in periglacial soil movements on alpine crestslopes. - Earth Surface Processes and Landforms, 30: 41-58. DOI: 10.1002/esp.1125.

MatsuoKa, N. (2003): Contemporary permafrost and periglaciation in Asian high mountains: an overview. - Zeitschrift für Geomorphologie, NF, Suppl.-Bd. 130: 145-166.

Ministerium Für Bildung und Wissenschaft der RePUBLIK KASACHSTAN (2004): Geographisches Institut, unveröffentlichte Temperaturdaten.

Passmore, D., Harrison, S., Winchester, V., SeverSkiy, I. \& Pimankina, N.V. (2008): Late Holocene Debris Flows and Valley Floor Development in the Northern Zailiiskiy Alatau, Tien Shan Mountains, Kazakhstan. - Arctic, Antarctic, and Alpine Research, 40/3: 548-560. DOI: 10.1657/15230430(06-078)[PASSMORE]2.0.CO;2.
Romanovsky, V.E. \& Osterkamp, T.E. (2000): Effects of Unfrozen Water on Heat and Mass Transport Processes in the Active Layer and Permafrost. - Permafrost and Periglacial Processes, 11: 219-239. DOI:10.1002/1099$1530(200007 / 09) 11: 3<219:$ : A I D PPP352>3.0.CO;2-7.

Scheffer, F. \& Schachtschabel, P. (2002): Lehrbuch der Bodenkunde, 15. Aufl. - 593 S; Heidelberg (Spektrum).

SCHRÖDER, H., GunJA, A. \& Fickert, T. (1996): Vergleichende Periglazialmorphologie im zentralen Teil des nördlichen Tien-Shan. - Mitteilungen der Fränkischen Geographischen Gesellschaft, 43: 275 - 300 .

Schröder, H., Kokarev, A. \& Harrison, S. (2005): Rock glaciers in the northern Tien Shan, Kazakhstan: new data on movement rates and distribution. - Glacial Geology and Geomorphology, rp01/2005.

Troll, C. (1973): High Mountain belts between the Polar Caps and Equator. Their Definition and Lower Limit. - Arctic and Alpine Research, 5/3: A19-A27.

Veit, H., Stingl, H., Emmerich, K.-H. \& John, B. (1995): Zeitliche und räumliche Variabilität solifluidaler Prozesse und ihre Ursachen. - Zeitschrift für Geomorphologie, NF; Suppl.-Bd. 99: 107-122.

Williams, P. \& Smith, M. (1989): The Frozen Earth. Fundamentals of Geocryology. - 306 S.; Cambridge (Camb. Univ. Press). 